\title{
The effect of manual small incision cataract surgery on the intraocular pressure - A prospective study in south Kerala
}

\author{
Jasmine Mary Jacob ${ }^{1}$, Ann Divya Jacob ${ }^{2}$, Sheldon Goudinho ${ }^{3, *}$ \\ ${ }^{1}$ Professor and HOD, ${ }^{2}$ Junior Resident, ${ }^{3}$ Professor, Dept. of Ophthalmology, Dr. Somervelle Memorial CSI Medical College, \\ Karakonam, Kerala, India \\ *Corresponding Author: \\ Email: shelandjas@yahoo.com
}

\begin{abstract}
Aim: To study the effect of manual small incision cataract surgery (SICS) on the intraocular pressure (IOP) postoperatively at one week, three weeks and three months.

Materials and Methods: Prospective longitudinal study, conducted at Dr SMCSI College from January 2016 -May 2017. Sample size: 50 eyes. Patients who underwent uneventful SICS were included. Patients with pre-existing glaucoma, were excluded. Goldman applanation tonometer was used to measure IOP. Preoperative IOP was measured. All patients underwent manual SICS with PCIOL implantation by one surgeon. Postoperative IOP measurements were taken at one week, three week, and three month postoperatively. Analysis done using SPSS 16.0.

Results: The mean preoperative IOP was $15.16(\mathrm{SD}+/-2.566) \mathrm{mm} \mathrm{Hg}$. The mean one week postoperative IOP was 14.08 (SD +/2.320) $\mathrm{mm} \mathrm{Hg}$. The mean postoperative IOP at three weeks was 13.68 (SD +/- 2.343) $\mathrm{mm} \mathrm{Hg}$. The mean postoperative IOP at three month was 13.12 (SD +/- 2.496) $\mathrm{mm} \mathrm{Hg}$. Paired sample t-test showed $\mathrm{p}<0.001$ in all groups which was statistically significant. The final mean reduction in IOP postoperative at three months was $2.040 \mathrm{mmHg}$.
\end{abstract}

Conclusion: There was significant reduction in intraocular pressure after manual SICS.

Keywords: Cataract, Glaucoma, Intra ocular pressure, Small incision cataract surgery.

\section{Introduction}

Cataract is one of the leading cause of preventable blindness in the world. More than $74 \%$ of the adults aged 60 and above are diagnosed with cataract or have undergone cataract surgery. ${ }^{1}$ The Indian study of age related eye disease reported the prevalance of unoperated cataract in people aged $\geq 60$ was $58 \%$ in north India and $53 \%$ south India. ${ }^{2}$ Manual small incision cataract surgery and phacoemulsification with intraocular lens implantation are the types of catarct surgery commonly done. Manual SICS is a safe and very cost effective way to tackle the huge backlog of cataract in a developing country like India. Early rehabilitation,easy learning curve and the feasibility with almost all types of cataract has made manual SICS popular. There are about 11.2 million people aged 40 and more affected by glaucoma in our country. ${ }^{3}$ Glaucoma can be primarily of two types open angle and closed angle. Howewer intraocular pressure is the only modifiable risk factor in glaucoma irrespective of the type of glaucoma. All the treatmment modalities in glaucoma aim at reducing the intraocular pressure. In the past few years emphasis was given on postoperative astigmatism after manual SICS, postoperative intraocular pressure change is a less studied topic. The concomitance of glaucoma and cataract coupled with the fact that the only modifiable risk factor in glaucoma management is intraocular pressure, has led to this study. There are many patients who are diagnosed with cataract as well as glaucoma but are lost on follow up or non compliant with the antiglaucoma drugs. There are many studies that describe the effect of phacoemulsification on IOP, ${ }^{4-6}$ but there is paucity of information on the effect of manual small incision cataract surgery on IOP in the Southern part of our country. Long term follow up analysis on the postoperative intraocular pressure and angle grading after manual SICS merits attention in order to prognosticate patients with glaucoma or ocular hypertension.

\section{Materials and Methods}

An Institutional based prospective longitudinal study conducted at the Department of Ophthalmology at Dr. SMCSI Medical College from January 2016 to May 2017. A sample size of 50 eyes were calculated. Informed consent was obtained from all study subjects. This study adhered to the tenets of the declaration of Helsinki and the research protocol was approved by the institutional Ethics Committee. All patients diagnosed to have cataract willing to undergo Manual SICS were included in the study.

Exclusion Criteria: Patients with pre-operative IOP more than $21 \mathrm{~mm} \mathrm{Hg}$, Angle pathology, peripheral anterior synechiae, corneal opacity, congenital, juvenile or complicated cataract, history of glaucoma or ocular surgeries in the past, any intraoperative complications were excluded from the study. All patients underwent a comprehensive ophthalmic examination. Lens Opacities Classification System (LOCS) III was used to grade the type and density of cataract. A calibrated Goldmann applanation tonometer was used to measure IOP. IOP was repeated and recorded for the second time after a time interval of 30 minutes. If the two measurements 
differed by more than $3 \mathrm{~mm} \mathrm{Hg}$, a third measurement was taken. Every attempt was made to keep subsequent IOP measurements within a 4 -hour time window to reduce diurnal variation. Preoperative IOP was considered as the mean of 2 IOP or the meridian of 3 IOP measurements. Postoperative IOP was recorded at one week, 3 weeks and 3 months follow up period. All patients in this study underwent standard manual small incision cataract surgery with posterior capsular intraocular lens implantation under regional anaesthesia by one surgeon.

Analysis: Analysis was done using SPSS 16.0. All qualitative variables were calculated using percentage and all quantitative variables were calculated using mean and standard deviation. The intraocular pressure change preoperatively and postoperatively were compared using paired sample t-test. $\mathrm{p}<0.05$ was considered for statistical significance.

\section{Results}

The mean age of patients was 65.6 years $(\mathrm{SD} \pm$ 9.467); minimum age was 50 years and maximum age was 89 years. Among 50 patients, 20 patients $(60 \%)$ were Males and 30 patients $(40 \%)$ were females. There were $25(50 \%)$ right eyes and $25(50 \%)$ left eyes among the study population. The mean preoperative IOP was $15.16(\mathrm{SD} \pm 2.566) \mathrm{mmHg}$ (Fig. 1)

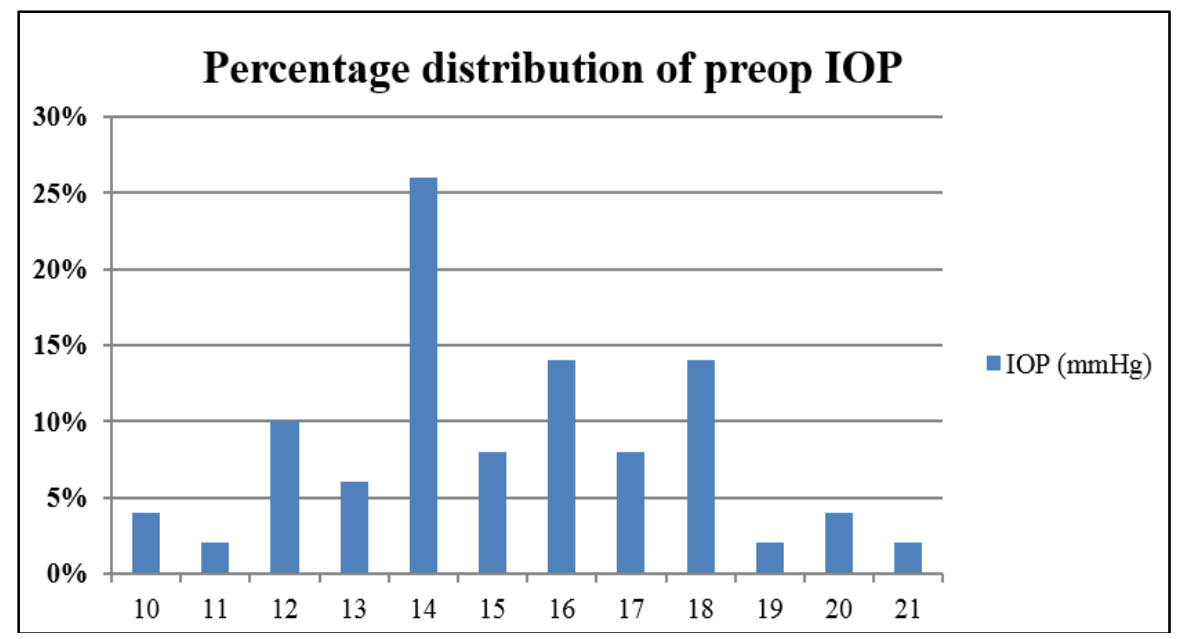

Fig. 1: Percentage distribution of preoperative IOP

The mean IOP at postoperative 1 week was 14.08(SD \pm 2.320$) \mathrm{mm} \mathrm{Hg}$. The mean change in IOP

was $1.080(\mathrm{SD} \pm 1.759) \mathrm{mm} \mathrm{Hg}$ which was statistically significant.

Table 1: Comparision between preoperative IOP and IOP at 1 week postoperative

\begin{tabular}{|l|c|c|c|}
\hline Intraocular pressure & Mean & SD & \\
\hline IOP(preop) & 15.16 & 2.566 & \multirow{2}{*}{ P $<0.001 *$} \\
\hline IOP(one week postop) & 14.08 & 2.320 & \multirow{2}{*}{} \\
\hline IOP(preop) -IOP(one week postop) & 1.080 & 1.759 & \\
*paired t-test
\end{tabular}

The mean IOP at 3 weeks postoperative period was $13.68(\mathrm{SD} \pm 2.343) \mathrm{mm} \mathrm{Hg}$. The mean change in IOP was $1.480(\mathrm{SD} \pm 2.102)$ which was statistically significant

Table 2: Comparision between Preoperative IOP and IOP at 3 week postoperative

\begin{tabular}{|l|c|c|c|}
\hline \multicolumn{1}{|c|}{ Intraocular pressure } & Mean & SD & \\
\hline IOP (preop) & 15.16 & 2.566 & \multirow{2}{*}{$\mathrm{P}<0.001 *$} \\
\hline IOP(3weeks postop) & 13.68 & 2.343 & \multirow{2}{*}{} \\
\hline IOP preop) -IOP (3weekspostop) & 1.480 & 2.102 & \\
*paired t-test
\end{tabular}

The mean IOP at 3 months postoperative period was 13.12 (SD \pm 2.496$) \mathrm{mmHg}$. The mean change in intraocular pressure at 3 months was 2.040 which was statistically significant. 
Table 3: Comparision between Preoperative IOP and IOP at 3 months postoperatively

\begin{tabular}{|l|c|c|c|}
\hline \multicolumn{1}{|c|}{ Intraocular pressure } & Mean & SD & \\
\cline { 1 - 3 } IOP(preop) & 15.16 & 2.566 & \multirow{2}{*}{$*$} \\
\cline { 1 - 3 } IOP(3monthspostop) & 13.12 & 2.496 & \multirow{2}{*}{$* 0.001$} \\
\hline IOP (preop)-IOP (3monthspostop) & 2.040 & 2.416 & \\
\hline
\end{tabular}

*paired t-test

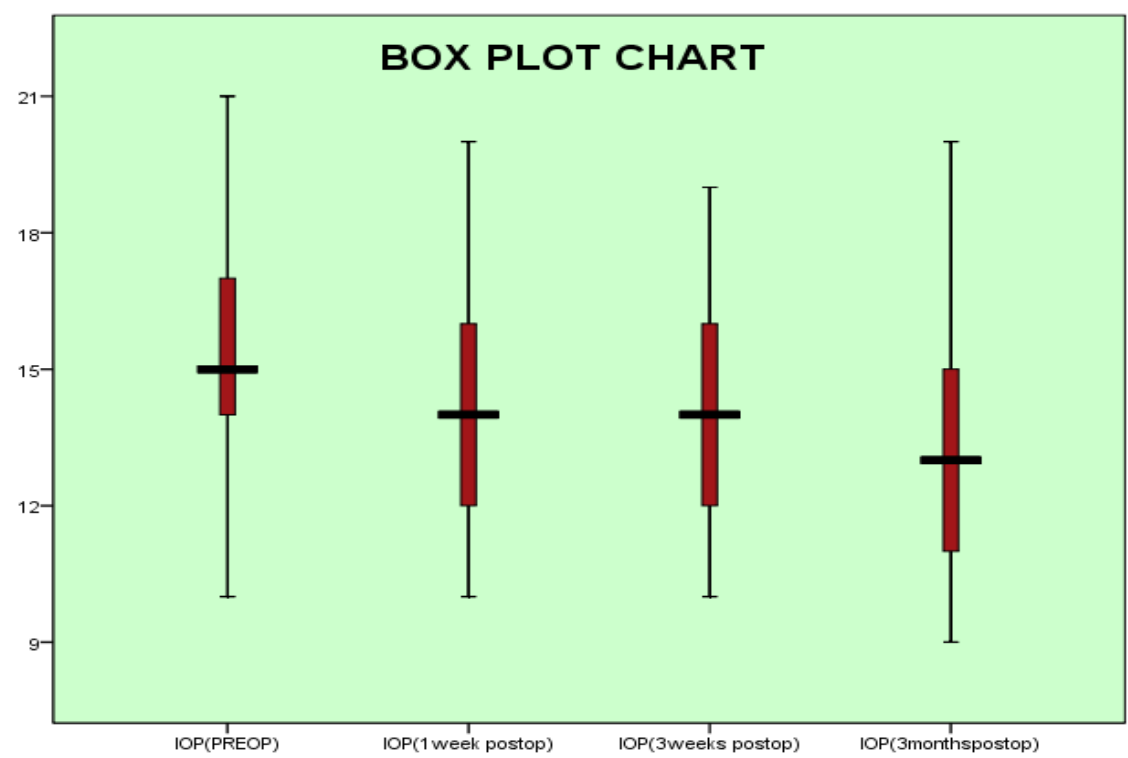

Fig. 2: Box plot chart representing the $25^{\text {th }}, 50^{\text {th }}, 7^{\text {th }}$ percentile of the IOP preoperative, 1 week postoperative, 3 weeks postoperative and 3 months postoperatively

\section{Discussion}

In this study the changes in the intraocular pressure were compared preoperatively and postoperatively at 1 week, 3 week, 3 months after manual SICS. The IOP reduction seen at 3 months of follow up was 2.040 (SD $\pm 2.416) \mathrm{mmHg}$ which was statistically significant $(p<0.001)$. Out of 50 eyes that were studied 3 eyes had no change in the intraocular pressure, 9 eyes had rise in intraocular pressure and 38 eyes showed reduction in intraocular pressure at 3 months of follow up. Sengupta et $\mathrm{al}^{7}$ compared the intraocular pressure reduction after phacoemulsification with manual small incision cataract surgery and they concluded that both phacoemulsification and manual SICS led to significant and equivalent IOP reduction after 6 months of surgery. $\Delta$ IOP $=2.7+/-2.9 \mathrm{mmHg}$ was seen in phacoemulsification subgroup and $\Delta \mathrm{IOP}=2.6+/-2.6$ $\mathrm{mmHg} ; \mathrm{P}<0.70$ ) was seen in manual SICS subgroup. This was similar to the change in IOP (2.040) in our study.

The exact pathophysiology of the IOP reduction is not clearly understood. Wang et $\mathrm{al}^{8}$ used normal and glaucomatous trabecular meshwork cell culture lines in vitro, and showed that ultrasound energy produced by the phaco probe propagated through a fluid medium induced a stress response which in turn lead to production of interleukin 1 , which may be involved in the reduction in IOP that occurs after phacoemulsification. The pressure reduction after manual SICS cannot be explained by this phenomenon since no ultrasound energy is produced during surgery. Murray johnstones ${ }^{9}$ mechanical pump theory explains why pressure reduction occurs after manual SICS. Cataract extraction with IOL implantation repositions the anterior lens capsule rearward, thereby allowing the ciliary body to rotate rearward, pivoting around the axis of the sclera spur. Cataract surgery does not damage the pump rather, it allows the pump to re-expand, and regain its earlier function allowing a better facility of outflow.

There are certain factors that are not taken into consideration which could have influenced the results of our study such as diurnal variation of IOP, lack of corneal hysteresis measurement, postoperative uveitis, steroid responders and shorter duration of follow up. Certain eyes have greater sclera rigidity which could have prevented expansion of the anterior chamber volume and angle structures after cataract extraction. Corneal hysteresis thus playing a major role in these eyes. All patients are put on postoperative medications including steroids, some of them can be steroid responders. IOP rise in 9 patients in our study can be explained by this phenomenon though longer duration of follow up period is required such cases. Postoperative uveitis can increase as well as decrease the intraocular pressures. 


\section{Conclusion}

There is significant reduction in the intraocular pressure at 3 months after manual SICS. Cataract surgery provides the patient better visual acuity, clarity of media for posterior segment evaluation, better visual field testing. Possibly, manual SICS can also aid patients with ocular hypertension by reducing their intraocular pressure.

\section{References}

1. Murthy GV, Gupta SK, Maraini G, Camparini M, Price GM, Dherani M, et al. Prevalence of lens opacities in North India: the INDEYE feasibility study. Investigative Ophthalmology \& Visual Science. 2007;48(1):88-95.

2. Vashist P, Talwar B, Gogoi M, Maraini G, Camparini M, Ravindran RD, et al. Prevalence of Cataract in an Older Population in India: The India Study of Age-related Eye Disease. Ophthalmology. 2011;118(2-19):272-8.e2.

3. George R, Ve RS, Vijaya L. Glaucoma in India: estimated burden of disease. Journal of Glaucoma. 2010;19(6):391-7.

4. Lagreze WD, Bomer TG, Funk J. Effect of surgical technique on the increase in intraocular pressure after cataract extraction. Ophthalmic Surg Lasers. 1996;27(3):169-73.

5. Liu X-Q, Zhu H-Y, Su J, Hao X-J. Effects of phacoemulsification on intraocular pressure and anterior chamber depth. Experimental and Therapeutic Medicine. 2013;5(2):507-10.
6. Euswas A, Warrasak S. Intraocular pressure control following phacoemulsification in patients with chronic angle closure glaucoma. Journal of the Medical Association of Thailand = Chotmaihet thangphaet . 2005;88 Suppl 9:S121-5.

7. Sengupta S, Venkatesh R, Krishnamurthy P, Nath M, Mashruwala A, Ramulu PY, et al. Intraocular Pressure Reduction after Phacoemulsification versus Manual Small-Incision Cataract Surgery: A Randomized Controlled Trial. Ophthalmology. 2016;123(8):1695-703.

8. Wang N, Chintala SK, Fini ME, Schuman JS. Ultrasound activates the TM ELAM-1/IL-1/NF-kappaB response: a potential mechanism for intraocular pressure reduction after phacoemulsification. Investigative Ophthalmology \& Visual Science. 2003;44(5):1977-81.

9. Johnstone MA, Grant WM. Pressure-Dependent Changes in Structures of the Aqueous Outflow System of Human and Monkey Eyes. American Journal of Ophthalmology. 1973;75(3):365-83.

How to cite this article: Jacob JM, Jacob AD, Goudinho S. The effect of manual small incision cataract surgery on the intraocular pressure - A prospective study in south Kerala. Ind J Clin Exp Ophthalmol. 2018;4(3):409-412. 